\title{
La obesidad y su relación con otros factores de riesgo cardiovascular en una población de estudiantes universitarios del centro de Bogotá, D.C.
}

\author{
Celis Luís Gustavo ${ }^{1}$, Obregón María Clara ${ }^{2}$, Ayala Jaime ${ }^{1}$, Blackburn Natalia ${ }^{1}$, Montaño Gina Sofia $^{1}$, \\ Gómez Mariana ${ }^{1}$, Páez Verónica ${ }^{1}$, Emmanuelli Juan $^{1}$, Domínguez María Teresa ${ }^{1}$, \\ Giratá Vilma ${ }^{3}$, Almonacid Carmen Cecilia ${ }^{3}$.
}

Facultad de Medicina. Universidad de La Sabana.

Programa de Administración de Instituciones de Servicio. Universidad de La Sabana.

Programa de Bacteriología. Universidad Colegio Mayor de Cundinamarca.

Correspondencia: luis.celis@unisabana.edu.co

Recibido: 07/05/2013 Aceptado: 02/07/2013

\section{Resumen}

La obesidad y sus complicaciones constituyen una de las principales pandemias a nivel mundial, en especial la Diabetes tipo 2 que está asociada a la resistencia a la insulina. El propósito de este trabajo es describir la relación existente entre la obesidad y otros de factores de riesgo cardiovascular en una población de estudiantes universitarios del centro de Bogotá. Previo consentimiento informado, se procedió a la medición de la composición corporal, a través de la toma de: pliegue tricipital y supraescapular mediante adipometro, perímetro de cintura, Índice de Masa Corporal, Masa Magra, Masa Grasa y porcentaje de grasa entre otras. También se realizaron tomas de muestras de sangre para determinar glucemia, colesterol total, triglicéridos y lipoproteínas, cuya relación permite el cálculo del índice aterogénico.

De una muestra final de 76 estudiantes, el 15,5\% presenta sobrepeso; el 76,5 \% está dentro de rangos considerados normales; y el 8,0\% presenta bajo peso. En lo referente a los datos por sexo, se encontró que un 5,5\% de hombres y un 19,0\% de las mujeres presentaban sobrepeso y un $5,5 \%$ de los hombres y un $8,6 \%$ de las mujeres presentaban bajo peso, en ningún caso hubo personas obesas. El perímetro de cintura varió entre 68 y $97 \mathrm{~cm}$ en hombres y entre 61 y $92 \mathrm{~cm}$ en mujeres, finalmente el porcentaje de grasa estuvo entre 27,6 a 30,7 en hombres y entre 11,0 a 36,9 en mujeres. Teniendo en cuenta los resultados obtenidos, es importante promover en nuestra población una cultura de hábitos saludables, caracterizada por una alimentación saludable y una mayor actividad física; también se recomienda, especialmente durante los primeros años de vida, ya que es en esta etapa donde se adquieren o instauran gran parte de los mismos y que en muchos casos permanecerán toda la vida.

Palabras clave: composición corporal, índice de masa corporal, obesidad, perfil lipídico, riesgo cardiovascular. 


\section{Obesity and its relationship with other cardiovascular risk factors in a population of university students in downtown Bogotá}

Obesity and its complications are one of the major pandemics at the global level, especially type 2 diabetes that is associated with insulin resistance. The purpose of this paper is to describe the relationship between obesity and other cardiovascular risk factors of university students in downtown Bogota. After obtaining an informed consent, we proceeded to the measurement of the following body parts: triceps skinfold and suprascapular through adipometer, waist circumference, body mass index, lean mass, fat mass and fat percentage among others. Blood samples were also taken to determine blood glucose, total cholesterol, triglycerides, and lipoproteins, whose relationship allows the calculation of the atherogenic index.

From a final sample of 76 students, $15.5 \%$ was overweight; $76.5 \%$ was considered within normal ranges; and the $8.0 \%$ was underweight. In regard to the data by sex, it was found that a $5.5 \%$ of men and $19.0 \%$ of women were overweight and $5.5 \%$ of men and $8.6 \%$ of the women had low weight, there were no obese people in any case. The waist perimeter varied between 68 and $97 \mathrm{~cm}$ in men and between 61 and $92 \mathrm{~cm}$ in women, finally the fat percentage was between 27.6 and 30.7 in men and from 11.0 to 36.9 in women. Taking into account the results obtained, it is important to promote in our population a culture of healthy habits, characterized by a healthy diet and increased physical activity; it is also recommended, especially during the first years of life, as it is in this stage where the majority of these habits are acquired and will last for life.

Key Words: body composition, body mass index, cardiovascular risk, obesity, lipid profile.

\section{Introducción}

La obesidad y sus complicaciones constituyen una de las principales pandemias del Siglo XXI. En la actualidad, a nivel mundial como en Colombia, la obesidad se ha convertido en un problema de salud pública y un factor de riesgo para enfermedades como diabetes, cáncer de próstata y colón, enfermedades cardiovasculares e hipertensión (1).

La obesidad constituye un problema de salud pública tanto en Estados Unidos como en el resto del mundo, igualmente se presenta en los países propensos a la desnutrición. A nivel mundial 1,1 billones de adultos sufren de sobrepeso y de ellos 312 millones son obesos, igualmente esta enfermedad está afectando a la población infantil, estimándose que hay unos 155 millones de niños con obesidad o sobrepeso. Una de las complicaciones derivadas de la obesidad es la diabetes, se estima que para el año 2003 se incrementó en Estados Unidos y Canadá el número de casos con diabetes en $72 \%$ mientras que en América Latina y el Caribe este incremento fue del orden del 148\% (2).

En el caso de las enfermedades cardiovasculares, éstas constituyen la primera causa de mortalidad y una de las principales fuentes de invalidez en los países desarrollados y en gran parte de los países en vía de desarrollo. Su etiología es variada y multifactorial, incluye factores fisiológicos, bioquímicos y ambientales que interactúan de manera sinérgica en el inicio y desarrollo de las enfermedades cardiovasculares $(3,4)$. 
Este tipo de patologías afecta en mayor proporción a individuos adultos, pero actualmente se han venido presentado un mayor número de casos de niños y adolescentes con sobrepeso y obesidad, lo cual ha traído como consecuencia la aparición, en etapas tempranas, de complicaciones en las enfermedades cardiovasculares $(5,6)$. El propósito de este trabajo es describir la relación existente entre la obesidad y otros de factores de riesgo cardiovascular en una población de estudiantes universitarios del centro de Bogotá.

\section{Metodología}

Previo aviso del consentimiento informado se procedió a la medición de la composición corporal, a través de la toma de pliegue tricipital mediante adipometro, perímetro de cintura, Índice de Masa Corporal (IMC), Masa Magra, Masa Grasa y porcentaje de grasa, entre otras. Esto se hizo con impedanciómetro Tanita Electronics Model TBF 310A (Tanita Corporation).

También se realizaron tomas de muestras de sangre con ayuno previo de 12 horas, para realizar determinaciones de glucemia, colesterol total, triglicéridos y lipoproteínas (HDL y LDL), mediante el autoanalizador Mindray BS-200 (Annar Diagnostica Import SAS).

\section{Resultados}

Los resultados obtenidos nos indican que, de una muestra final de 76 estudiantes, el $15.5 \%$ presenta sobrepeso, el 76,5 \% normopeso y el $8 \%$ bajo peso. En lo referente a los datos por sexo se encontró que un $5.5 \%$ de hombres y un 19\% de las mujeres presentaban sobrepeso y un $5.5 \%$ de los hombres y un $8.6 \%$ de las mujeres presentaban bajo peso, en ningún caso hubo personas obesas (figura 1).

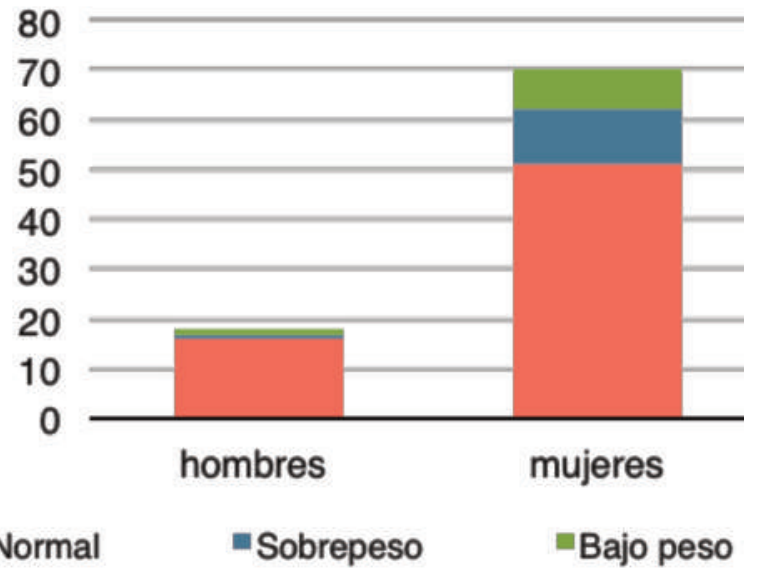

Figura 1. Distribución del estado nutricional de acuerdo al Índice de Masa Corporal (IMC) por sexo

El perímetro de cintura varió entre $68 \mathrm{~cm}$ y $97 \mathrm{~cm}$ en hombres y entre $61 \mathrm{~cm}$ y $92 \mathrm{~cm}$ en mujeres, finalmente el \% de grasa estuvo entre 27.6 a 30.7 en hombres y con un intervalo de 11 a 36.9 en las mujeres. Estos resultados señalan que, en la muestra estudiada, las mujeres presentan una mayor tendencia al sobrepeso que los hombres, lo cual también se ve reflejado en un mayor $\%$ de grasa corporal, coincidiendo estos datos con los registrados en la Encuesta Nacional de Nutrición del Año 2010 (7). Es importante resaltar los casos de normopeso que se encontraban cercanos a un IMC de 18,5, al igual que los de bajo peso, ya que estos podrían reflejar un grado de desnutrición, con la implicación que este aspecto puede tener en el proceso cognitivo.

En lo que respecta a la química sanguínea, un $33 \%$ de los casos estudiados presentan colesterolemia asociada con bajos niveles de HDL (33\%) y altos niveles de LDL (22\%). De todos los casos estudiados, 7 de los individuos analizados (4 hombres y 3 mujeres) presentan alto riesgo para desarrollar Síndrome Metabólico, de hecho uno de ellos evidenció un riesgo aterogénico (LDL/ HDL) de 10.6, con respecto a un valor de referencia de 3.22 para mujeres. 


\section{Discusión}

Estos hallazgos nos sugieren que es importante promover en nuestra población una cultura de hábitos saludables, caracterizada por una alimentación saludable y una mayor actividad física, que constituyen dos de las principales alternativas para la prevención de la obesidad.

En la actualidad, se reconoce la importancia que tienen los estilos de vida sobre el nivel de salud de la población. Uno de los aspectos que influyen de manera decisiva sobre la salud es la conducta alimentaria. Diversos trabajos han puesto de manifiesta la importancia y la elevada prevalencia en la población de hábitos alimentarios inadecuados. Según los expertos, todos los grupos de edad se ven afectados por conductas alimentarias no saludables, pero es en los primeros años de la vida donde estas cuestiones son especialmente relevantes, ya que en estos momentos es cuando se adquieren e instauran gran parte de los hábitos, que en muchos casos permanecerán durante toda la vida (8-12).

Sin embargo, es importante señalar que una alteración del perfil lipídico, como en el caso del colesterol y los triglicéridos, pueden deberse a causas genéticas, por lo que sería importante en futuro estudios para revisar si hay antecedentes de enfermedades metabólicas como la hipercolesterolemia familiar (13).

\section{Referencias}

1. Fu, M., Sun, T., Bookout, A. L., Downes, M., Yu, R. T., Evans, R. M. and Mangelsdorf, D. J. A Nuclear Receptor Atlas: 3T3-L1 Adipogenesis Molecular Endocrinology. 2005; 19(10):2437-2450.

2. Hossain, P., Kawar, B. y El Nahas, M. Obesity and Diabetes in the Developing World-Growing Challenge. Endocrine Reviews. 2006; Vol. 356 (3):213-215.
3. Lipska K, Sylaja PN, Sarma PS, Thankappan KR, Kutty VR, Vasan Rs et al. Risk factors for acute ischaemic stroke in Young adults in South India. J Neurol Neurosurg Psychiatry. 2007; 78: 959-963.

4. Sánchez-Contreras M, Moreno-Gómez GA, Marín-Grisales ME, Gracia Ortiz LH. Factores de Riesgo Cardiovascular en Jóvenes. Rev. salud pública. 2009; 11(1): 110-122.

5. Grisales H, Caicedo B, Serna C, Uribe D. Causas de mortalidad en jóvenes y su contribución al cambio en la esperanza de vida: Cali, 1989-1999. Colomb Med. 2005; 36(2): 85-93.

6. Campo JE, Reyes JJ, Ortiz C, Quintero L, Herrera JA. Niveles de presión arterial y de estrés psicosocial en estudiantes de la Facultad de Salud, Universidad del Valle. Cali, Colombia, 20032004. Colomb Med. 2006; 37(2 suppl. 1): 21-25.

7. Encuesta Nacional de Nutrición 2010 (Resumen Ejecutivo). Instituto Colombiano de Bienestar Familiar. Recuperado 28 de Marzo de 2011. http://www.corazonesresponsables.org/Resumen_Ejecutivo_ENSIN_2010.pdf

8. Suárez Herrera JC, Navarro Vázquez FJ, . Serra L, Armas A, Arancet J. Nivel de conocimientos, actitudes y hábitos sobre alimentación y nutrición en escolares de las Palmas de Gran Canaria Rev Esp Nutr Comunitaria. 2002;8(1-2):7-18.

9. Rojas Alcántara P, Pina Roche F, Bastida Pozuelo F, Muñoz Pérez G, González Quijano A, Santesteban de Mingo M. Alimentación de los escolares. Un diagnóstico de salud. Revista Rol de enfermería. 1990; 13:33-4.

10. Gómez López J, López Giménez MR. Hábitos alimentarios en adolescentes: Resultados de un estudio transversal en el municipio de Madrid según diferentes grupos de edad. Alimentación, Nutrición y Salud. 1996; 3(4):69-71.

11. Albaina Landaluce E, Díez Andrés MA, Sedano Garage MA. Hábitos dietéticos de una población obesa. Enfermería Clínica. 1996;6(1):18-22.

12. Martí-Henneberg C. Nutrición en Pediatría. Tratado de Pediatría. Vol 1. Barcelona: Espaxs S.A., 1988; 88:537-540.

13. Atienza G. Hipercolesterolemia familiar: evaluación del diagnóstico genético mediante micromatrices de ADN. Santiago de Compostela: Consellería de Sanidade, Axencia de Avaliación de Tecnoloxías Sanitarias de Galicia, avalia-t; 2006. Serie Avaliación de tecnoloxías. Consultas Técnicas: CT2006/02. 